\title{
EXTRAPOLATION IN THE SCALE OF GENERALIZED REVERSE HÖLDER WEIGHTS
}

\author{
THERESA C. ANDERSON, DAVID CRUZ-URIBE, OFS, AND KABE MOEN \\ ABSTRACT. We develop a theory of extrapolation for weights that satisfy a gener- \\ alized reverse Hölder inequality in the scale of Orlicz spaces. This extends previous \\ results by Auscher and Martell [2] on limited range extrapolation. We then provide \\ several applications of our extrapolation techniques. These applications include \\ new and known two weight inequalities for linear and bilinear operators.
}

\section{INTRODUCTION}

The theory of extrapolation is a powerful technique in harmonic analysis: in a nutshell, it shows that norm inequalities for an operator on the weighted Lebesgue space $L^{p}(w)$, for any $1<p<\infty$ and $w$ in the Muckenhoupt weight class $A_{p}$, are all a consequence of such an inequality being true for a single value of $p$. Extrapolation was introduced by Rubio de Francia [30] more than thirty years ago; since then it has been refined and developed in a number of directions, including applications to Banach functions spaces, bilinear inequalities and two weight norm inequalities. We refer the reader to [5] for a more detailed discussion of extrapolation and its history.

In its classic form, extrapolation depends on the weight $w$ being in the Muckenhoupt $A_{p}$ class, $1<p<\infty$ :

$$
[w]_{A_{p}}=\sup _{Q}\left(f_{Q} w d x\right)\left(f_{Q} w^{1-p^{\prime}} d x\right)^{p-1}<\infty,
$$

where the supremum is taken over all cubes $Q$ with sides parallel to the coordinate axes. More recently, Auscher and Martell [2] introduced a "limited range" version of extrapolation that depends on both the $A_{p}$ class of the weight and its reverse Hölder class $R H_{s}, 1<s<\infty$. We say $w \in R H_{s}$ if

$$
[w]_{R H_{s}}=\sup _{Q}\left(f_{Q} w d x\right)^{-1}\left(f_{Q} w^{s} d x\right)^{\frac{1}{s}}<\infty,
$$

Date: June 14, 2017.

2000 Mathematics Subject Classification. 42B20, 42B25.

The first author was supported by NSF grant DMS-1502464 and by an NSF Graduate Student Fellowship, and completed part of this work while in residence at MSRI in Spring 2017. The second author is supported by NSF Grant 1362425 and research funds from the Dean of the College of Arts \& Sciences, the University of Alabama. The third author is supported by Simons Collaborative Grant for Mathematicians 16-0427. The authors would like to thank J. M. Martell for helpful discussions which revealed a subtle mistake in an early version of this paper. 
where the supremum is taken over all cubes $Q$. (For a precise statement of their result, see Theorem 2.2 below.)

As an immediate consequence of their result we prove an extrapolation theorem that only depends on the reverse Hölder class of the weight. We state it here; for precise definitions of the notation used, please see Section 2.

Theorem 1.1. Let $0<q_{0}<\infty$ and $\mathcal{F}=\{(f, g)\}$ be a family of pairs of measurable functions. Suppose there exists $p_{0}$ with $0<p_{0} \leq q_{0}$ such that for all $w \in R H_{\left(\frac{q_{0}}{p_{0}}\right)^{\prime}}$,

$$
\|f\|_{L^{p_{0}(w)}} \leq C\|g\|_{L^{p_{0}(w)}} \quad(f, g) \in \mathcal{F} .
$$

Then for all $p, 0<p<q_{0}$, and $w \in R H_{\left(\frac{q_{0}}{p}\right)^{\prime}}$ we have

$$
\|f\|_{L^{p}(w)} \leq C\|g\|_{L^{p}(w)} \quad \forall(f, g) \in \mathcal{F} .
$$

The goal of this paper is to prove a generalization of Theorem 1.1 to a larger class of weights. To state our results we use the theory of Young functions and Orlicz norms; for precise definitions, see Section 3. Given a Young function $\Psi$, we say $w \in R H_{\Psi}$ if

$$
\sup _{Q}\left(f_{Q} w d x\right)^{-1}\|w\|_{\Psi, Q}<\infty
$$

where again the supremum is taken over all cubes $Q$. This class was first introduced by Harboure et al. [13] with a different but essentially equivalent definition. When $\Psi(t)=t^{s}$, this reduces to the class $R H_{s}$ defined above, and as we will show below, these weights also satisfy the classical reverse Hölder inequality. Intuitively, they differ in that they capture different behavior at different values of the range of the weight. For instance, if $\Psi$ is the "oscillatory" Young function

$$
\Psi(t)=t^{s+a \sin \left(\log \log \left(e^{e}+t\right)\right)}, \quad 0<a<s-1,
$$

then depending on its size, on some cubes $w \in R H_{\Psi}$ behaves like a weight in $R H_{s+a}$ and on others like a weight in $R H_{s-a}$. Using these weights we prove the following extrapolation theorem. The class $B_{r}$ is a growth condition on Young functions: see Section 3 below for a definition.

Theorem 1.2. Given $0<p_{0}<q_{0}$, suppose that for a fixed $\Psi_{0} \in B_{\left(\frac{q_{0}}{p_{0}}\right)^{\prime}}$ and all $w \in R H_{\Psi_{0}}$,

$$
\|f\|_{L^{p_{0}(w)}} \lesssim\|g\|_{L^{p_{0}(w)}}, \quad(f, g) \in \mathcal{F}
$$

Define the Young function $\Psi$ by $\Psi_{0}(t)=\Psi\left(t^{r}\right)$ with $r=\frac{\left(q_{0} / p_{0}\right)^{\prime}}{\left(q_{0} / p\right)^{\prime}}<1$. If $p_{0}<p<q_{0}$ and $w \in R H_{\Psi}$, then we have that

$$
\|f\|_{L^{p}(w)} \lesssim\|g\|_{L^{p}(w)}, \quad(f, g) \in \mathcal{F} .
$$

We actually prove a more general result when $p=q_{0}$ and also when $p_{0}=q_{0}$; see Theorem 3.5 below.

To illustrate the utility of our extrapolation results, we give applications to the study of two weight norm inequalities for linear and bilinear Calderón-Zygmund singular integral operators, and to the theory of one weight inequalities for the bilinear 
fractional integral operator. Two weight norm inequalities have been studied for many years by a number of authors. In recent years this problem has received renewed attention because of its close connection to the so-called " $A_{2}$ conjecture" for singular integral operators that was proved by Hytönen [15]. In the decade of work that led to the proof of this result, it became clear that in order to get the desired sharp constant estimate, the problem had to be treated as a two-weight problem, with the $A_{p}$ condition used only once at some key step.

The techniques used, particularly the dyadic sparse operators that were introduced by Lerner [20], have been applied to the study of " $A_{p}$ bump" conditions for two-weight norm inequalities. This approach to generalizing the two-weight $A_{p}$ condition was first introduced by Neugebauer [26] but was systematically developed by Pérez [27, 28] (see also [5] and [1]). The following result was first conjectured by Pérez and the second author [7] and finally proved by Lerner [20].

Theorem 1.3. Suppose $1<p<\infty$ and $\Phi$ and $\Psi$ are Young functions such that $\bar{\Phi} \in B_{p^{\prime}}$ and $\bar{\Psi} \in B_{p}$. If $(u, v)$ is a pair of weights that satisfies

$$
\sup _{Q}\|u\|_{\Phi, Q}\left\|v^{-1}\right\|_{\Psi, Q}<\infty
$$

then given any Calderón-Zygmund singular integral operator $T$,

$$
\|(T f) u\|_{L^{p}} \lesssim\|f v\|_{L^{p}}
$$

Below (see Theorem 4.2) we give a new proof of this result using extrapolation. Moreover, we prove a slight generalization, proving that $T$ satisfies a two weight, Coifman-Fefferman type inequality:

$$
\|(T f) u\|_{L^{p}} \lesssim\left\|M_{\bar{\Psi}}(f v)\right\|_{L^{p}} .
$$

We also extend Theorem 1.3 to the bilinear setting, proving the analogous result for bilinear Calderón-Zygmund singular integral operators. These are the natural generalization of the linear operators, and have been considered by a number of authors: see $[12,17]$. One weight norm inequalities were characterized by $[11,19]$. Our results in the two weight case are new. The exact condition required depends on whether $p>1$ or $1 / 2<p \leq 1$ : see Theorems 4.5 and 4.6.

Finally, we consider weighted norm inequalities for the bilinear fractional integral operator

$$
B I_{\alpha}(f, g)(x)=\int_{\mathbb{R}^{n}} \frac{f(x-y) g(x+y)}{|y|^{n-\alpha}} d y
$$

This operator is the fractional analog of the bilinear Hilbert transform; for weighted norm inequalities and a history of this operator, see $[8,14,25]$. The corresponding maximal operator is

$$
B M_{\alpha}(f, g)(x)=\sup _{r>0} \frac{1}{(2 r)^{n-\alpha}} \int_{[-r, r]^{n}}|f(x-y) g(x+y)| d y .
$$

We also recall the less singular bilinear fractional operators:

$$
\mathcal{I}_{\alpha}(f, g)(x)=\int_{\mathbb{R}^{2 n}} \frac{f(y) g(z)}{(|x-y|+|x-z|)^{2 n-\alpha}} d y d z, \quad 0<\alpha<2 n,
$$


and the associated maximal operator

$$
\mathcal{M}_{\alpha}(f, g)(x)=\sup _{Q \ni x}|Q|^{\frac{\alpha}{n}} f_{Q}|f(y)| d y \cdot f_{Q}|g(z)| d z \quad 0 \leq \alpha<2 n .
$$

These operators are the fractional operators corresponding to bilinear CZOs.

As an application of our extrapolation techniques, we are able to prove a CoifmanFefferman type inequality relating $B I_{\alpha}$ to the the less singular bilinear maximal operator, $\mathcal{M}_{\alpha}$. Our result improves one that was first proved by the third author in $[25]$.

The remainder of this paper is organized as follows. In Section 2 we give some preliminary information about Muckenhoupt weights and extrapolation, and then prove Theorem 1.1. In Section 3 we give the necessary background information on Young functions and Orlicz spaces, and then prove two extrapolation theorems. The first one is an unweighted extrapolation used in our applications, Theorem 3.4, and the second is Theorem 3.5, a generalization of Theorem 1.2. Though some of the proof of Theorem 3.4 overlaps with Theorem 3.5, we include the proof for two reasons. First, it is more general due to the range of $p$; second, the proof makes clear the main ideas while avoiding the technicalities that arise in the weighted case. Finally, in Section 4 we prove our applications in Theorems 4.2, 4.5, 4.6, and 4.7.

Throughout this paper, $n$ will denote the dimension of the space $\mathbb{R}^{n}$. If we write $A \lesssim B$, we mean $A \leq C B$ for some constant $C ; A \approx B$ means $A \lesssim B$ And $B \lesssim A$. Whether implicit or explicit, unless otherwise specified the constants may depend on the dimension $n, p$, the weights and the operator being studied, and can change from line to line.

Remark 1.4. As we were completing this paper, we learned that Theorem 1.1 was discovered independently by Martell and Prisuelos [23].

\section{2. $A_{p}, R H_{s}$ WEIGHTS AND EXTRAPOLATION}

Preliminaries about weights. Hereafter, by a weight we mean a non-negative, locally integrable function. We begin with a few preliminary facts about $A_{p}$ and $R H_{s}$ weights we will need in this and the following section. Beyond the $A_{p}$ and $R H_{s}$ classes defined above, we define three additional weight classes. We say that a weight $w \in A_{1}$ if

$$
[w]_{A_{1}}=\sup _{Q}\left(f_{Q} w d x\right) \operatorname{ess} \sup _{x \in Q}\left(w^{-1}(x)\right)<\infty,
$$

where the supremum is taken over all cubes $Q$. Recall that the $A_{p}$ classes are nested: for all $q>p>1, A_{1} \subset A_{p} \subset A_{q}$. Analogously, we define $w \in R H_{\infty}$ if

$$
[w]_{R H_{\infty}}=\sup _{Q}\left(\operatorname{esssup}_{x \in Q} w(x)\right)\left(f_{Q} w d x\right)^{-1}<\infty
$$


where the supremum is taken over all cubes $Q$; then for all $r<s<\infty, R H_{\infty} \subset$ $R H_{s} \subset R H_{r}$. Finally, we let $A_{\infty}$ denote the union of the $A_{p}$ classes:

$$
A_{\infty}=\bigcup_{1<p<\infty} A_{p}
$$

There is a close connection between $A_{p}$ weights and the Hardy-Littlewood maximal operator. Given $f \in L_{l o c}^{1}$, define

$$
M f(x)=\sup _{Q} f_{Q}|f| d y \cdot \chi_{Q}(x)
$$

where the supremum is taken over all cubes $Q$.

For proofs of the results in the following lemma, see $[6,9,10]$.

Lemma 2.1. Given a weight w:

(a) $w \in A_{\infty}$ if and only if there exists $s>1$ such that $w \in R H_{s}$;

(b) $w \in A_{\infty}$ if and only if there exist constants $0<\alpha, \beta<1$ such that given any cube $Q$ and $E \subset Q$ with $|E|<\alpha|Q|, w(E)<\beta w(Q)$;

(c) $w \in R H_{s}$ for some $1<s<\infty$ if and only if $w^{s} \in A_{\infty}$;

(d) if $w \in R H_{\infty}$, then $w^{s} \in R H_{\infty}$ for all $s>0$;

(e) given $p>1$, if $w \in A_{1}$ then $w^{1-p^{\prime}} \in R H_{\infty} \cap A_{p}$, and if $w \in R H_{\infty} \cap A_{p}$ then $w^{1-p^{\prime}} \in A_{1}$

(f) for all $0<r<1$, the function $(M w)^{r} \in A_{1}$.

Extrapolation. While a major application of extrapolation is to prove norm inequalities for operators, it can be applied much more broadly if it is stated in terms of pairs of functions. We follow the formulation used in [5]. Hereafter, $\mathcal{F}=\{(f, g)\}$ will denote a family of pairs of non-negative, measurable functions that are not identically 0 . Given a fixed family $\mathcal{F}$ and some weighted space $L^{p}(w)$, if we write

$$
\|f\|_{L^{p}(w)} \lesssim\|g\|_{L^{p}(w)}, \quad(f, g) \in \mathcal{F}
$$

then we mean that this inequality holds for all pairs $(f, g)$ for which the lefthand term in the inequality is finite. (This assumption assures that in the underlying proofs, it is possible to estimate the norm by duality.) The constant $C$ can depend on the $A_{p}$ and/or $R H_{s}$ characteristic of $w$, and on $s$ and $p$, but it cannot depend on the weight $w$ itself.

In practice, to prove weighted norm inequalities for an operator $T$, it suffices to consider a family of pairs of functions of the form $(|T f|,|f|)$, where $f$ is taken from some suitably chosen dense family of functions (e.g., $f \in L_{c}^{\infty}$ ). In order to get the norm finiteness of the first term, we can replace $|T f|$ by $\min (|T f|, N) \chi_{B(0, N)}$ and then take the limit as $N \rightarrow \infty$.

We can now prove Theorem 1.1. As we noted in the Introduction, this result is a consequence of the limited range extrapolation theorem of Auscher and Martell [2, Theorem 4.9]. 
Theorem 2.2. Given $0<s_{0}<q_{0}<\infty$ and a family $\mathcal{F}=\{(f, g)\}$, suppose there exists $s_{0} \leq p_{0} \leq q_{0}$ such that for all $w \in A_{\frac{p_{0}}{s_{0}}} \cap R H_{\left(\frac{q_{0}}{p_{0}}\right)^{\prime}}$,

$$
\|f\|_{L^{p_{0}(w)}} \lesssim\|g\|_{L^{p_{0}}(w)} \quad(f, g) \in \mathcal{F} .
$$

Then for all $s_{0}<p<q_{0}$ and $w \in A_{\frac{p}{s_{0}}} \cap R H_{\left(\frac{q_{0}}{p}\right)^{\prime}}$,

$$
\|f\|_{L^{p}(w)} \lesssim\|g\|_{L^{p}(w)} \quad(f, g) \in \mathcal{F} .
$$

Proof of Theorem 1.1. Fix $0<p<q_{0}$ and $w \in R H_{\left(\frac{q_{0}}{p}\right)^{\prime}}$; we will show that

$$
\|f\|_{L^{p}(w)} \lesssim\|g\|_{L^{p}(w)}, \quad(f, g) \in \mathcal{F} .
$$

By Lemma 2.1(a), we can fix $q$ sufficiently large so that $w \in A_{q} \cap R H_{\left(\frac{q_{0}}{p}\right)^{\prime}}$. Fix $0<s_{0}<\min \left(\frac{p}{q}, p_{0}\right)$. Then by assumption, for all $v \in A_{\frac{p_{0}}{s_{0}}} \cap R H_{\left(\frac{q_{0}}{p_{0}}\right)^{\prime}} \subset R H_{\left(\frac{q_{0}}{p_{0}}\right)^{\prime}}$,

$$
\|f\|_{L^{p_{0}(v)}} \lesssim\|g\|_{L^{p_{0}(v)}} \quad(f, g) \in \mathcal{F}
$$

Therefore, the hypotheses of Theorem 2.2 are satisfied, and since we have that $w \in$ $A_{\frac{p}{s_{0}}} \cap R H_{\left(\frac{q_{0}}{p}\right)^{\prime}}$, the desired inequality holds.

\section{Orlicz Reverse Hölder Extrapolation}

Young functions and Orlicz norms. Here we gather the basic properties of Young functions and Orlicz norms that we will use. We follow [5]; for proofs see, for example, [29].

A Young function $\Phi:[0, \infty) \rightarrow[0, \infty)$ is a convex, increasing, continuous function such that $\Phi(0)=0$ and

$$
\lim _{t \rightarrow \infty} \frac{\Phi(t)}{t}=\infty
$$

Young functions are sometimes normalized so that $\Phi(1)=1$; doing so simplifies the constants that appear below.

Given a Young function $\Phi$, the complementary Young function $\bar{\Phi}$ is defined by

$$
\bar{\Phi}(s)=\sup _{t}\{s t-\Phi(t)\}
$$

If $\Phi(t)=t^{p}, p>1$, then $\bar{\Phi}(t)=t^{p^{\prime}}$. More generally,

$$
\Phi^{-1}(t) \bar{\Phi}^{-1}(t) \approx t
$$

Another important example are Orlicz functions of the form $\Phi(t)=t^{p} \log (e+t)^{p-1+\delta}$, $\delta>0$. In this case we have that

$$
\bar{\Phi}(t) \approx \frac{t^{p^{\prime}}}{\log (e+t)^{1+\left(p^{\prime}-1\right) \delta}} .
$$

Given a Young function $\Phi$ and a cube $Q$, we define the localized Orlicz norm

$$
\|f\|_{\Phi, Q}=\inf \left\{\lambda>0: f_{Q} \Phi\left(\frac{|f(x)|}{\lambda}\right) d x \leq 1\right\} .
$$


If $\Phi(t)=t^{p}$, then we get the localized Lebesgue norm:

$$
\|f\|_{\Phi, Q}=\left(f_{Q}|f|^{p} d x\right)^{\frac{1}{p}} .
$$

These norms form an increasing scale: more precisely, given Young functions $\Phi$ and $\Psi$, if $\Phi(t) \lesssim \Psi(c t)$, then

$$
\|f\|_{\Phi, Q} \lesssim\|f\|_{\Psi, Q}
$$

with a constant independent of $Q$.

This norm satisfies versions of Hölder's inequality: given a Young function $\Phi$ and a cube $Q$,

$$
f_{Q}|f g| d x \leq 2\|f\|_{\Phi, Q}\|g\|_{\bar{\Phi}, Q}
$$

More generally, given Young functions $\Phi, \Psi, \Theta$ such that $\Phi^{-1}(t) \Psi(t)^{-1} \lesssim \Theta^{-1}(t)$, then

$$
\|f g\|_{\Theta, Q} \leq 2\|f\|_{\Phi, Q}\|g\|_{\Psi, Q} .
$$

We now define two growth conditions on Young functions. The first bounds a Young function from below. Given a Young function $\Psi$ and $a>1$, we say that $\Psi$ is an $a$-Young function if $\Psi_{a}(t)=\Psi\left(t^{\frac{1}{a}}\right)$ is a Young function. In this case, $t^{a} \lesssim \Psi(t)$ for large $t$. The second condition bounds a Young function from above. Given $1<p<\infty$, a Young function $\Phi$ satisfies the $B_{p}$ condition, denoted by $\Phi \in B_{p}$, if

$$
\int_{1}^{\infty} \frac{\Psi(t)}{t^{p}} \frac{d t}{t}<\infty .
$$

In this case we have $\Psi(t) \lesssim t^{p}$ for large $t$. This condition was introduced by Pérez [28] to study the Orlicz maximal operators

$$
M_{\Psi} f(x)=\sup _{Q \ni x}\|f\|_{\Psi, Q}
$$

He proved the following $L^{p}$ estimate.

Theorem 3.1. For all $1<p<\infty,\left\|M_{\Psi} f\right\|_{L^{p}} \lesssim\|f\|_{L^{p}}$ if and only if $\Psi \in B_{p}$.

Remark 3.2. In [28] the statement of this result contained the further hypothesis that $\Psi$ was doubling; however, this was shown to be superfluous by Liu and Luque [22] (see also [1]).

Finally, we have that the generalized reverse Hölder class $R H_{\Phi}$ is contained in the scale of $A_{p}$ weights. More precisely, we have the following lemma that was proved in [13] in a slightly different form. For the convenience of the reader we repeat the short proof.

Lemma 3.3. Given any Young function $\Phi$, if $w \in R H_{\Phi}$, then $w \in A_{\infty}$.

Proof. Fix a cube $Q$ and a measurable set $E \subset Q$. We first estimate the norm $\left\|\chi_{E}\right\|_{\bar{\Phi}, Q}$. Fix $\lambda>0$ such that

$$
f_{Q} \bar{\Phi}\left(\frac{\chi_{E}(x)}{\lambda}\right) d x=1 .
$$


Then we have that

$$
\left\|\chi_{E}\right\|_{\bar{\Phi}, Q}=\lambda=\bar{\Phi}^{-1}\left(\frac{|Q|}{|E|}\right)^{-1}
$$

Since $\Phi^{-1}(t) \bar{\Phi}^{-1}(t) \approx t$, we get that

$$
\left\|\chi_{E}\right\|_{\bar{\Phi}, Q} \simeq \frac{|E|}{|Q|} \Phi^{-1}\left(\frac{|Q|}{|E|}\right) .
$$

We now estimate as follows:

$$
w(E)=|Q| f_{Q} w \chi_{E} d x \lesssim|Q|\|w\|_{\Phi, Q}\|\| \chi_{E} \|_{\bar{\Phi}, Q} \lesssim w(Q) \frac{|E|}{|Q|} \Phi^{-1}\left(\frac{|Q|}{|E|}\right) .
$$

Since $\Phi$ is a Young function, we have that

$$
\lim _{s \rightarrow \infty} \frac{s}{\Phi^{-1}(s)}=\lim _{t \rightarrow \infty} \frac{\Phi(t)}{t}=\infty .
$$

Therefore, we can find $0<\alpha, \beta<1$ such that if $|E| /|Q|<\alpha$, then $w(E) / w(Q)<\beta$. Then by Lemma 2.1(b), $w \in A_{\infty}$.

Extrapolation with generalized reverse Hölder weights. We can now state and prove our main extrapolation theorems. Our first result yields unweighted inequalities.

Theorem 3.4. Given $p_{0}<q_{0}$ and $\Psi_{0} \in B_{\left(\frac{q_{0}}{p_{0}}\right)^{\prime}}$, suppose that for all $w \in R H_{\Psi_{0}}$,

$$
\|f\|_{L^{p_{0}(w)}} \leq C\|g\|_{L^{p_{0}(w)}}, \quad(f, g) \in \mathcal{F} .
$$

Then for all $0<p \leq q_{0}$,

$$
\|f\|_{L^{p}} \leq C\|g\|_{L^{p}}, \quad(f, g) \in \mathcal{F}
$$

If $p_{0}=q_{0}$, the same conclusion holds if we assume (3.1) holds whenever $w \in R H_{\infty}$.

Theorem 3.4 is actually a consequence of Theorem 1.1. Since $\Psi_{0} \in B_{\left(\frac{q_{0}}{p_{0}}\right)^{\prime}}, \Psi_{0}(t) \lesssim$ $t^{\left(\frac{q_{0}}{p_{0}}\right)^{\prime}}$ for $t \geq 1$. Therefore, if $w \in R H_{\left(\frac{q_{0}}{p_{0}}\right)^{\prime}}$,

$$
\|w\|_{\Psi_{0}, Q} \lesssim\|w\|_{\left(\frac{q_{0}}{p_{0}}\right)^{\prime}, Q} \lesssim\|w\|_{1, Q}
$$

and so $w \in R H_{\Psi_{0}}$. Thus (3.1) implies that (1.1) holds, and so by Theorem 1.1, for $0<p<q_{0},(1.2)$ holds. If we take $w=1$ we get (3.2).

Despite this, here we give a direct proof. We do so for two reasons. First, our proof is ultimately simpler, since it avoids limited range extrapolation which itself is nontrivial to prove. Second, as we noted in the Introduction, our proof makes clear the main ideas that will be used in the proof of Theorem 3.5, which is not a consequence of Theorem 2.2 .

Proof. We first consider the case $p_{0}<p \leq q_{0}$ (the case $p=p_{0}$ is obvious by taking $w=1)$. By duality we have that

$$
\|f\|_{L^{p}}=\left\||f|^{p_{0}}\right\|_{L^{\frac{p}{p_{0}}}}^{\frac{1}{p_{0}}}=\left(\int|f|^{p_{0}} h d x\right)^{\frac{1}{p_{0}}}
$$


for some $h \in L^{\left(\frac{p}{p_{0}}\right)^{\prime}}$ with norm one. Moreover, since the $B_{p}$ classes are nested we have

$$
\Psi_{0} \in B_{\left(\frac{q_{0}}{p_{0}}\right)^{\prime}} \subset B_{\left(\frac{p}{p_{0}}\right)^{\prime}}
$$

since $p \leq q_{0}$. Hence

$$
M_{\Psi_{0}}: L^{\left(\frac{p}{p_{0}}\right)^{\prime}} \rightarrow L^{\left(\frac{p}{p_{0}}\right)^{\prime}} .
$$

We now define a Rubio de Francia iteration algorithm:

$$
\mathcal{R} h=\sum_{k=0}^{\infty} \frac{M_{\Psi_{0}}^{k} h}{2^{k}\left\|M_{\Psi_{0}}\right\|_{L^{\left(\frac{p}{p_{0}}\right)^{\prime}}}^{k}},
$$

where $M_{\Psi_{0}}^{0} h=|h|$. The operator $\mathcal{R}$ satisfies the following properties:

(a) $|h| \leq \mathcal{R} h$,

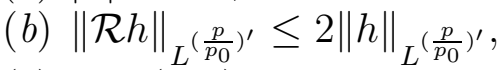

(c) $M_{\Psi_{0}}(\mathcal{R} h) \leq C \mathcal{R} h$,

(d) $\mathcal{R} h \in R H_{\Psi_{0}}$.

The first three points are standard: cf. [5]. To prove the final point, fix a cube $Q$. Then we have that

$$
\|\mathcal{R} h\|_{\Psi_{0}, Q} \leq f_{Q} M_{\Psi_{0}}(\mathcal{R} h) \leq C f_{Q} \mathcal{R} h
$$

We can now estimate as follows:

$$
\begin{aligned}
\left(\int|f|^{p_{0}} h d x\right)^{\frac{1}{p_{0}}} & \leq\left(\int|f|^{p_{0}} \mathcal{R} h d x\right)^{\frac{1}{p_{0}}} \\
& \leq C\left(\int|g|^{p_{0}} \mathcal{R} h d x\right)^{\frac{1}{p_{0}}} \quad\left(\text { since } \mathcal{R} h \in R H_{\Psi_{0}}\right) \\
& \leq C\left(\int|g|^{p}\right)^{\frac{1}{p}}\left(\int \mathcal{R} h^{\left(\frac{p}{p_{0}}\right)^{\prime}} d x\right)^{\frac{1}{p_{0}\left(\frac{p}{p_{0}}\right)^{\prime}}} \\
& \leq 2^{\frac{1}{p_{0}}} C\left(\int|g|^{p} d x\right)^{\frac{1}{p}} .
\end{aligned}
$$

We now consider the case when $0<p<p_{0} \leq q_{0}$. This case is much simpler and only relies on the maximal operator. Fix $r>\frac{1}{p}$ and define $H=M\left(g^{\frac{1}{r}}\right)^{\frac{p r}{\left(p_{0} / p\right)^{\prime}}}$. Then $H^{-p_{0} / p}=M\left(g^{\frac{1}{r}}\right)^{-a}$ for $a>0$, and so by Lemma $2.1(\mathrm{e}, \mathrm{f}), H^{-p_{0} / p} \in R H_{\infty} \subset R H_{\Psi_{0}}$.

We can now estimate as follows: by our hypothesis and since the maximal operator is bounded on $L^{p r}$,

$$
\begin{aligned}
\|f\|_{L^{p}}^{p} & =\int_{\mathbb{R}^{n}} f^{p} H^{-1} H d x \\
& \leq\left(\int_{\mathbb{R}^{n}} f^{p_{0}} H^{-p_{0} / p} d x\right)^{p / p_{0}}\left(\int_{\mathbb{R}^{n}} H^{\left(p_{0} / p\right)^{\prime}} d x\right)^{1 /\left(p_{0} / p\right)^{\prime}} \\
& \lesssim\left(\int_{\mathbb{R}^{n}} g^{p_{0}} H^{-p_{0} / p} d x\right)^{p / p_{0}}\left(\int_{\mathbb{R}^{n}} M\left(g^{\frac{1}{r}}\right)^{p r} d x\right)^{1 /\left(p_{0} / p\right)^{\prime}}
\end{aligned}
$$




$$
\begin{aligned}
& \leq\left(\int_{\mathbb{R}^{n}} g^{p_{0}}\left(g^{\frac{1}{r}}\right)^{r\left(p-p_{0}\right)} d x\right)^{p / p_{0}}\left(\int_{\mathbb{R}^{n}} g^{p} d x\right)^{1 /\left(p_{0} / p\right)^{\prime}} \\
& =\int_{\mathbb{R}^{n}} g^{p} d x .
\end{aligned}
$$

Now we state and prove Theorem 3.5, the more general version of Theorem-1.2.

Theorem 3.5. Given $0<p_{0}<q_{0}$, suppose that for a fixed $\Psi_{0} \in B_{\left(\frac{q_{0}}{p_{0}}\right)^{\prime}}$ and all $w \in R H_{\Psi_{0}}$,

$$
\|f\|_{L^{p_{0}(w)}} \lesssim\|g\|_{L^{p_{0}(w)}}, \quad(f, g) \in \mathcal{F}
$$

If $p_{0}=q_{0}$, suppose (3.3) holds for any $w \in R H_{\infty}$. If either of the following hold:

(a) $p_{0}<p<q_{0}$ and $w \in R H_{\Psi}$, where $\Psi$ is defined by $\Psi_{0}(t)=\Psi\left(t^{r}\right)$ with $r=$ $\frac{\left(q_{0} / p_{0}\right)^{\prime}}{\left(q_{0} / p\right)^{\prime}}<1$

(b) $p=q_{0}$ and $w \in R H_{\infty}$;

then we have that

$$
\|f\|_{L^{p}(w)} \lesssim\|g\|_{L^{p}(w)}, \quad(f, g) \in \mathcal{F} .
$$

Remark 3.6. Notice that as $p$ gets close to $q_{0}$, then $\frac{1}{r} \rightarrow \infty$, so the second case is a natural endpoint condition.

Remark 3.7. In Theorem 3.5 we are not able to prove weighted inequalities in the range $0<p<p_{0} \leq q_{0}$ analogous to the unweighted inequalities in Theorem 3.4. Our proof in the unweighted case does not extend to the weighted setting. This problem seems to be much more subtle and will require new techniques.

Proof. The proof follows the same outline as the proof of Theorem 3.4, and we refer to that proof for some details that are the same. We consider each case in turn.

First suppose that $p_{0}<p<q_{0}$; by duality there exists $h \in L^{\left(\frac{p}{p_{0}}\right)^{\prime}},\|h\|_{L^{\left(\frac{p}{p_{0}}\right)^{\prime}}}=1$, such that

$$
\|f\|_{L^{p}(w)}^{p_{0}}=\left(\int_{\mathbb{R}^{n}} f^{p_{0} \frac{p}{p_{0}}} w^{\frac{p_{0}}{p} \frac{p}{p_{0}}} d x\right)^{\frac{p_{0}}{p}}=\int_{\mathbb{R}^{n}} f^{p_{0}} w^{\frac{p_{0}}{p}} h d x .
$$

Since $\Psi_{0}(t)=\Psi\left(t^{r}\right), \Psi \in B_{\left(\frac{q_{0}}{p}\right)^{\prime}}$ : by a change of variables we have that

$$
\int_{1}^{\infty} \frac{\Psi(t)}{t^{\left(\frac{q_{0}}{p}\right)^{\prime}}} \frac{d t}{t} \approx \int_{1}^{\infty} \frac{\Psi_{0}(t)}{t^{\left(\frac{q_{0}}{p_{0}}\right)^{\prime}}} \frac{d t}{t}<\infty
$$

the last inequality holds by our assumption that $\Psi_{0} \in B_{\left(\frac{q_{0}}{p_{0}}\right)^{\prime}}$.

Now suppose that we have a non-negative function $H$ that satisfies the following conditions:

(a) $h \leq H$

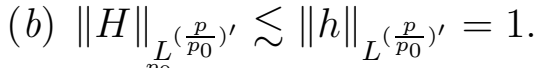

(c) $H w^{\frac{p_{0}}{p}} \in R H_{\Psi_{0}}$; 
Then by our hypothesis and the properties of $H$ we can estimate as follows:

$$
\begin{aligned}
\int_{\mathbb{R}^{n}} f^{p_{0}} w^{\frac{p_{0}}{p}} h d x & \leq \int_{\mathbb{R}^{n}} f^{p_{0}} H w^{\frac{p_{0}}{p}} d x \\
& \lesssim \int_{\mathbb{R}^{n}} g^{p_{0}} H w^{\frac{p_{0}}{p}} d x \\
& \leq\left\|g^{p_{0}} w^{\frac{p_{0}}{p}}\right\|_{L^{\frac{p}{p_{0}}}}\|H\|_{L^{\left(\frac{p}{p_{0}}\right)^{\prime}}} \\
& \leq\|g\|_{L^{p}(w)}^{p_{0}}
\end{aligned}
$$

Therefore, to complete the argument for this case we need to construct a function $H$ with the desired properties. We first construct two auxiliary Young functions. Let $C(t)=\Psi\left(t^{\frac{p}{p_{0}}}\right)$. We claim that $w^{\frac{p_{0}}{p}} \in R H_{C}$. Indeed, by Lemma 3.3, $w \in A_{\infty}$ and so by Lemma 2.1(c) we have that $w^{\frac{p_{0}}{p}} \in R H_{\frac{p}{p_{0}}}$. Therefore, by rescaling the norm, we have that

$$
\left\|w^{\frac{p_{0}}{p}}\right\|_{C, Q}=\|w\|_{\Psi, Q}^{\frac{p_{0}}{p}} \lesssim\|w\|_{1, Q}^{\frac{p_{0}}{p}} \lesssim\left\|w^{\frac{p_{0}}{p}}\right\|_{1, Q}
$$

Now define $s>0$ by

$$
\frac{1}{s}=\frac{1}{r}-\frac{p_{0}}{p}
$$

If $1<\frac{1}{s}<\frac{1}{r}, \Psi\left(t^{s}\right)=\Psi\left(\left(t^{r}\right)^{s / r}\right)=\Psi_{0}\left(t^{s / r}\right)$, and $s / r>1$ so $B(t)=\Psi\left(t^{s}\right)$ is a Young function; on the other hand, if $0<\frac{1}{s} \leq 1$ then $s \geq 1$ and $B$ is again a Young function. Moreover, in either case we have that $B \in B_{\left(\frac{p}{p_{0}}\right)^{\prime}}$ and hence $M_{B}$ is bounded on $L^{\left(\frac{p}{p_{0}}\right)^{\prime}}$. To see this, first note that

$$
\frac{1}{s}\left(\frac{p}{p_{0}}\right)^{\prime}=\left(\frac{q_{0}-p_{0}}{q_{0}-p}-\frac{p_{0}}{p}\right) \frac{p}{p-p_{0}}=\frac{q_{0}\left(p-p_{0}\right)}{p\left(q_{0}-p\right)} \frac{p}{p-p_{0}}=\frac{q_{0}}{q_{0}-p}=\left(\frac{q_{0}}{p}\right)^{\prime} .
$$

Then by a change of variables and the fact that $\Psi \in B_{\left(\frac{q_{0}}{p}\right)^{\prime}}$,

$$
\int_{1}^{\infty} \frac{B(t)}{t^{\left(\frac{p}{p_{0}}\right)^{\prime}}} \frac{d t}{t}=\int_{1}^{\infty} \frac{\Psi\left(t^{s}\right)}{t^{\left(\frac{p}{p_{0}}\right)^{\prime}}} \frac{d t}{t} \approx \int_{1}^{\infty} \frac{\Psi(t)}{t^{\left(\frac{q_{0}}{p}\right)^{\prime}}} \frac{d t}{t}<\infty .
$$

We can now define $H$ using a Rubio de Francia iteration algorithm:

$$
H=\mathcal{R} h=\sum_{k=0}^{\infty} \frac{M_{B}^{k} h}{2^{k}\left\|M_{B}\right\|_{L^{\left(\frac{p}{p_{0}}\right)^{\prime}}}^{k}} .
$$

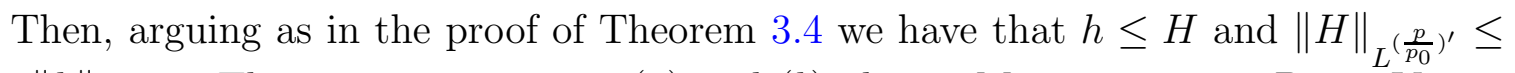

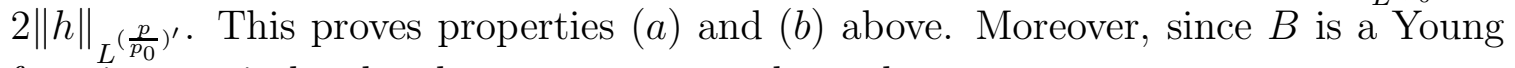
function, again by the above argument we have that

$$
M(\mathcal{R} h) \leq M_{B}(\mathcal{R} h) \lesssim \mathcal{R} h
$$

Thus $H \in A_{1} \cap R H_{B}$. By the definition of $B$ and $C$ we have that

$$
C^{-1}(t) B^{-1}(t)=\Psi^{-1}(t)^{\frac{p_{0}}{p}} \Psi^{-1}(t)^{\frac{1}{s}}=\Psi^{-1}(t)^{\frac{1}{r}}=\Psi_{0}^{-1}(t) .
$$


Therefore, by the generalized Hölder's inequality and the definition of $A_{1}$,

$$
\left\|H w^{\frac{p_{0}}{p}}\right\|_{\Psi_{0}, Q} \lesssim\|H\|_{B, Q}\left\|w^{\frac{p_{0}}{p}}\right\|_{C, Q} \lesssim\|H\|_{1, Q}\left\|w^{\frac{p_{0}}{p}}\right\|_{1, Q} \lesssim\left\|H w^{\frac{p_{0}}{p}}\right\|_{1, Q},
$$

which proves property $(c)$. This completes our proof when $p_{0}<p<q_{0}$.

The proof when $p=q_{0}$ is nearly the same as the previous case; here we describe the changes. Fix $w \in R H_{\infty}$, and let $\Psi_{0}$ be any Young function in $B_{\left(q_{0} / p_{0}\right)^{\prime}}$. Let $B=\Psi_{0}$ and define $H$ by (3.5). Then $H \in A_{1} \cap R H_{B}$ and satisfies properties $(a)$ and $(b)$ as before. To prove $(c)$ note first that by Lemma 2.1(d), $w^{\frac{p_{0}}{p}} \in R H_{\infty}$. By this, and then using that $H \in R H_{B}$ and then that $H \in A_{1}$,

$$
\left\|H w^{\frac{p_{0}}{p}}\right\|_{\Psi_{0}, Q} \lesssim\|H\|_{B, Q}\left\|w^{\frac{p_{0}}{p}}\right\|_{1, Q} \lesssim\|H\|_{1, Q}\left\|w^{\frac{p_{0}}{p}}\right\|_{1, Q} \lesssim\left\|H w^{\frac{p_{0}}{p}}\right\|_{1, Q} .
$$

Given this function $H$, the remainder of the proof goes through without change. This completes the proof.

\section{Applications}

In this section we give several applications of reverse Hölder extrapolation to prove weighted norm inequalities. In spirit, though not in detail, these applications are similar to those proved via $A_{\infty}$ extrapolation in [4].

Calderón-Zygmund operators. A Calderón-Zygmund kernel is a function $K(x, y)$ defined away from the diagonal $\{(x, y): x=y\}$ that satisfies

$$
|K(x, y)| \lesssim|x-y|^{-n}
$$

and

$$
\begin{aligned}
|K(x, y)-K(x, y+h)|+|K(x, y)-K(x+h, y)| & \\
& \leq C \frac{|h|^{\epsilon}}{|x-y|^{n+\epsilon}}, \quad|x-y|>2|h| .
\end{aligned}
$$

A Calderón-Zygmund operator $(\mathrm{CZO})$ is an $L^{2}$ bounded linear operator associated to a Calderón-Zygmund kernel $K$ such that the representation

$$
T f(x)=\int_{\mathbb{R}^{n}} K(x, y) f(y) d y
$$

holds for all $f \in L_{c}^{\infty}$ and $x \notin \operatorname{supp}(f)$.

To prove norm inequalities for CZOs we will use the theory of sparse operators over dyadic grids. The following is based on the seminal work of Lerner [20]; the pointwise estimates are due to Conde-Alonso and Rey [3] and Lacey [18]. (See also the recent monograph by Lerner and Nazarov [21], which uses a slightly different definition of a dyadic grid.)

By a dyadic grid $\mathcal{D}$ we mean a collection of cubes $\mathcal{D}=\bigcup_{k} \mathcal{D}_{k}$ in $\mathbb{R}^{n}$ that have the following properties:

(a) for each $k$, if $Q \in \mathcal{D}_{k}$, then $|Q|=2^{-k n}$;

(b) the cubes in $\mathcal{D}_{k}$ form a partition of $\mathbb{R}^{n}$;

(c) if $P, Q \in \mathcal{D}$, then $P \cap Q=\varnothing, P \subset Q$ or $Q \subset P$. 
Given a dyadic grid $\mathcal{D}$ we say a subfamily $\mathcal{S} \subset \mathcal{D}$ is sparse if for each $Q \in \mathcal{S}$

$$
\left|\bigcup_{\substack{Q^{\prime} \subset \mathcal{S} \\ Q^{\prime} \subsetneq Q}} Q^{\prime}\right| \leq \frac{1}{2}|Q|
$$

As a consequence, there exists $E_{Q} \subset Q$ such that the family $\left\{E_{Q}\right\}_{Q \in \mathcal{S}}$ is pairwise disjoint and there exists a uniform constant such that $|Q| \leq c\left|E_{Q}\right|$.

Given a dyadic grid $\mathcal{D}$ and a sparse family $\mathcal{S} \subset \mathcal{D}$, define a sparse operator by

$$
T^{\mathcal{S}} f(x)=\sum_{Q \in \mathcal{S}}\left(f_{Q} f d y\right) \chi_{Q}(x) .
$$

Sparse operators are positive, linear operators. Their importance is that CZOs can be dominated by them pointwise.

Theorem 4.1. Given a CZO operator $T$ and a function $f$, there exist $3^{n}$ dyadic grids $\left\{\mathcal{D}^{k}\right\}_{k=1}^{3^{n}}$ and sparse families $\mathcal{S}^{k} \subset \mathcal{D}^{k}$ such that

$$
|T f(x)| \lesssim \sum_{k=1}^{3^{n}} T^{\mathcal{S}^{k}}(|f|)(x)
$$

almost everywhere. The implicit constant depends on the dimension and the kernel $K$ associated to $T$.

Using sparse operators and reverse Hölder extrapolation, we can prove our generalization of Theorem 1.3.

Theorem 4.2. Let $T$ be a CZO, and fix $1<p<\infty$. Suppose $(u, v)$ is a pair of weights that satisfies

$$
\sup _{Q}\|u\|_{\Phi, Q}\left\|v^{-1}\right\|_{\Psi, Q}<\infty
$$

where $\bar{\Phi} \in B_{p^{\prime}}$ and $\Psi$ is any Young function. Then

$$
\|(T f) u\|_{L^{p}} \lesssim\left\|M_{\bar{\Psi}}(f v)\right\|_{L^{p}} .
$$

In particular, if $\bar{\Psi} \in B_{p}$, then

$$
\|(T f) u\|_{L^{p}} \lesssim\|f v\|_{L^{p}}
$$

Remark 4.3. By using results from [16, 18], Theorem 4.2 can be extended to singular integral operators that replace (4.1) with a weaker Dini continuity condition. Details are left to the interested reader.

Proof. When $\bar{\Psi} \in B_{p}$, (4.3) follows immediately from (4.2). To prove this inequality, by Theorem 4.1 it will suffice to prove it with $T$ replaced by a sparse operator $T^{\mathcal{S}}$ and with $f$ non-negative. By Theorem 3.4 with $q_{0}=p$ and $p_{0}=1$, it will suffice to show that if $w \in R H_{\bar{\Phi}}$, then

$$
\left\|\left(T^{\mathcal{S}} f\right) u\right\|_{L^{1}(w)} \lesssim\left\|M_{\bar{\Psi}}(f v)\right\|_{L^{1}(w)} .
$$


This inequality follows by a straightforward computation using the properties of a sparse family. We have that

$$
\int_{\mathbb{R}^{n}}\left(T^{\mathcal{S}} f\right) u w d x=\sum_{Q \in \mathcal{S}}\left(f_{Q} f d x\right)\left(f_{Q} u w d x\right)|Q| .
$$

Further, since by Lemma $3.3, w \in R H_{\bar{\Phi}} \subset A_{\infty}$,

$$
\|w\|_{\bar{\Phi}, Q}|Q| \leq[w]_{R H_{\bar{\Phi}}} w(Q) \leq C w\left(E_{Q}\right)
$$

Therefore, by the generalized Hölder's inequality,

$$
\begin{aligned}
\sum_{Q \in \mathcal{S}}\left(f_{Q} f d x\right)\left(f_{Q} u w d x\right)|Q| & \leq \sum_{Q \in \mathcal{S}}\|f v\|_{\bar{\Psi}, Q}\left\|v^{-1}\right\|_{\Psi, Q}\|u\|_{\Phi, Q}\|w\|_{\bar{\Phi}, Q}|Q| \\
& \lesssim \sum_{Q \in \mathcal{S}}\|f v\|_{\bar{\Psi}, Q}\|w\|_{\bar{\Phi}, Q}|Q| \\
& \lesssim \sum_{Q \in \mathcal{S}}\|f v\|_{\bar{\Psi}, Q} w\left(E_{Q}\right) \\
& \lesssim \int_{\mathbb{R}^{n}} M_{\bar{\Psi}}(f v) w d x .
\end{aligned}
$$

Bilinear Calderón-Zygmund operators. The results of the previous section extend naturally to the multilinear setting. A bilinear $\mathrm{CZO}$ is defined by the integral formula

$$
T(f, g)(x)=\int_{\mathbb{R}^{n}} K(x, y, z) f(y) g(z) d y d z \quad x \notin(\operatorname{supp} f) \cap(\operatorname{supp} g) .
$$

for $f, g \in L_{c}^{\infty}\left(\mathbb{R}^{n}\right)$ where $K$ is a bilinear Calderón-Zygmund kernel:

$$
|K(x, y, z)| \lesssim(|x-y|+|x-z|)^{-2 n}, \quad|\nabla K(x, y, z)| \lesssim(|x-y|+|x-z|)^{-2 n-1} .
$$

Bilinear CZOs can also be dominated pointwise by bilinear sparse operators. Again, given a dyadic grid $\mathcal{D}$ and a sparse family $\mathcal{S} \subset \mathcal{D}$, we define

$$
T^{\mathcal{S}}(f, g)(x)=\sum_{Q \in \mathcal{S}}\left(f_{Q} f d y\right)\left(f_{Q} g d y\right) \chi_{Q}(x) .
$$

The following estimate was proved in [3, 21].

Theorem 4.4. Given a bilinear $C Z O, T$ and functions $f, g$, there exist $3^{n}$ dyadic grids $\mathcal{D}_{k}$ and sparse families $\mathcal{S}_{k} \subset \mathcal{D}_{k}$ such that

$$
|T(f, g)(x)| \lesssim \sum_{k=1}^{3^{n}} T^{\mathcal{S}_{k}}(|f|,|g|)(x) .
$$


Given two Young functions $\Psi_{1}$ and $\Psi_{2}$, we define the bisublinear maximal function

$$
\mathcal{M}_{\Psi_{1}, \Psi_{2}}(f, g)=\sup _{Q \ni x}\|f\|_{\Psi_{1}, Q}\|g\|_{\Psi_{2}, Q}
$$

Clearly we have that $\mathcal{M}_{\Psi_{1}, \Psi_{2}}(f, g)(x) \leq M_{\Psi_{1}} f(x) M_{\Psi_{2}} g(x)$, so by Hölder's inequality, if $\Psi_{1} \in B_{p_{1}}$ and $\Psi_{2} \in B_{p_{2}}$, then $\mathcal{M}_{\Psi_{1}, \Psi_{2}}: L^{p_{1}} \times L^{p_{2}} \rightarrow L^{p}$. We can now state and prove the analog of Theorem 4.2 for bilinear CZOs. We get two results; in the first we assume $p>1$.

Theorem 4.5. Let $T$ be a bilinear CZO, fix $1<p_{1}, p_{2}<\infty$, and define $p=\frac{p_{1} p_{2}}{p_{1}+p_{2}}$. Suppose $p>1$ and,$\left(u, v_{1}, v_{2}\right)$ are weights that satisfy

$$
\sup _{Q}\|u\|_{\Phi, Q}\left\|v_{1}^{-1}\right\|_{\Psi_{1}, Q}\left\|v_{2}^{-1}\right\|_{\Psi_{2}, Q}<\infty
$$

where $\Phi$ is a Young function with $\bar{\Phi} \in B_{p^{\prime}}$ and $\Psi_{1}, \Psi_{2}$ are Young functions. Then

$$
\|T(f, g) u\|_{L^{p}} \lesssim\left\|M_{\bar{\Psi}_{1}, \bar{\Psi}_{2}}\left(f v_{1}, g v_{2}\right)\right\|_{L^{p}} .
$$

In particular, if $\bar{\Psi}_{1} \in B_{p_{1}}$ and $\bar{\Psi}_{2} \in B_{p_{2}}$, then

$$
\|T(f, g) u\|_{L^{p}} \lesssim\left\|f v_{1}\right\|_{L^{p_{1}}}\left\|g v_{2}\right\|_{L^{p_{2}}} .
$$

Proof. As in the proof of Theorem 4.2, it will suffice to prove the first inequality; the second is an immediate corollary. And again, it will suffice to prove this for a bilinear sparse operator $T^{\mathcal{S}}$ and non-negative $f, g$. By Theorem 3.4 with $q_{0}=p$ and $p_{0}=1$ we only need to prove a weighted $L^{1}$ inequality.

Fix $w \in R H_{\bar{\Phi}}$; then we can essentially repeat the previous argument:

$$
\begin{aligned}
\int_{\mathbb{R}^{n}} T^{\mathcal{S}}(f, g) w u d x & =\sum_{Q \in \mathcal{S}}\left(f_{Q} f d x\right)\left(f_{Q} g d x\right)\left(f_{Q} u w d x\right)|Q| \\
& \leq \sum_{Q \in \mathcal{S}}\left\|f v_{1}\right\|_{\bar{\Psi}_{1}, Q}\left\|g v_{2}\right\|_{\bar{\Psi}_{2}, Q}\left\|v_{1}^{-1}\right\|_{\Psi_{1}, Q}\left\|v_{2}^{-1}\right\|_{\Psi_{2}, Q}\|u\|_{\Phi, Q}\|w\|_{\bar{\Phi}, Q}|Q| \\
& \lesssim \sum_{Q \in \mathcal{S}}\left\|f v_{1}\right\|_{\bar{\Psi}_{1}, Q}\left\|g v_{2}\right\|_{\bar{\Psi}_{2}, Q}\|w\|_{\bar{\Phi}, Q}|Q| \\
& \lesssim \sum_{Q \in \mathcal{S}}\|f v\|_{\bar{\Psi}_{1}, Q}\left\|g v_{2}\right\|_{\bar{\Psi}_{2}, Q} w\left(E_{Q}\right) \\
& \lesssim \int_{\mathbb{R}^{n}} M_{\bar{\Psi}_{1}, \bar{\Psi}_{2}}\left(f v_{1}, g v_{2}\right) w d x .
\end{aligned}
$$

Surprisingly, when $p \leq 1$ we do not need an Orlicz bump on the weight $u$ : it suffices to take the localized $L^{p}$ norm. 
Theorem 4.6. Let $T$ be a bilinear CZO, fix $1<p_{1}, p_{2}<\infty$, and define $p=\frac{p_{1} p_{2}}{p_{1}+p_{2}}$. Suppose $p \leq 1$ and $\left(u, v_{1}, v_{2}\right)$ are weights that satisfy

$$
\sup _{Q}\left(f_{Q} u^{p} d x\right)^{\frac{1}{p}}\left\|v_{1}^{-1}\right\|_{\Psi_{1}, Q}\left\|v_{2}^{-1}\right\|_{\Psi_{1}, Q}<\infty
$$

where $\Psi_{1}, \Psi_{2}$ are Young functions. Then

$$
\|T(f, g) u\|_{L^{p}} \lesssim\left\|M_{\bar{\Psi}_{1}, \bar{\Psi}_{2}}\left(f v_{1}, g v_{2}\right)\right\|_{L^{p}} .
$$

In particular, if $\bar{\Psi}_{1} \in B_{p_{1}}$ and $\bar{\Psi}_{2} \in B_{p_{2}}$ then

$$
\|T(f, g) u\|_{L^{p}} \lesssim\left\|f v_{1}\right\|_{L^{p_{1}}}\left\|g v_{2}\right\|_{L^{p_{2}}} .
$$

Proof. The proof is more straightforward than the proof of Theorem 4.5 since we do not need to use extrapolation. Again, we will prove it for a sparse bilinear operator $T^{\mathcal{S}}$ and a pair of non-negative functions $f, g$. Since $0<p \leq 1$, by convexity we have the pointwise inequality

$$
T^{S}(f, g)^{p} \leq \sum_{Q \in \mathcal{S}}\left[\left(f_{Q} f d x\right)\left(f_{Q} g d x\right)\right]^{p} \chi_{Q} .
$$

Therefore, proceeding as we did above,

$$
\begin{aligned}
\int_{\mathbb{R}^{n}}\left(T^{\mathcal{S}}(f, g) u\right)^{p} d x & \leq \sum_{Q \in \mathcal{S}}\left[\left(f_{Q} f d x\right)\left(f_{Q} g d x\right)\right]^{p}\left(f_{Q} u^{p} d x\right)|Q| \\
& \leq \sum_{Q \in \mathcal{S}}\left(\left\|f v_{1}\right\|_{\bar{\Psi}_{1}, Q}\left\|g v_{2}\right\|_{\bar{\Psi}_{2}, Q}\left\|v_{1}^{-1}\right\|_{\Psi_{1}, Q}\left\|v_{2}^{-1}\right\|_{\Psi_{2}, Q}\right)^{p}\left(f_{Q} u^{p} d x\right)|Q| \\
& \lesssim \sum_{Q \in \mathcal{S}}\left(\left\|f v_{1}\right\|_{\bar{\Psi}_{1}, Q}\left\|g v_{2}\right\|_{\bar{\Psi}_{2}, Q}\right)^{p}|Q| \\
& \lesssim \sum_{Q \in \mathcal{S}}\left(\left\|f v_{1}\right\|_{\bar{\Psi}_{1}, Q}\left\|g v_{2}\right\|_{\bar{\Psi}_{2}, Q}\right)^{p}\left|E_{Q}\right| \\
& \lesssim \int_{\mathbb{R}^{n}} M_{\bar{\Psi}_{1}, \bar{\Psi}_{2}}\left(f v_{1}, g v_{2}\right)^{p} d x
\end{aligned}
$$

Bilinear fractional integral operators. Recall from the Introduction that, given $0<\alpha<n$, we define the bilinear fractional integral operator

$$
B I_{\alpha}(f, g)(x)=\int_{\mathbb{R}^{n}} \frac{f(x-y) g(x+y)}{|y|^{n-\alpha}} d y
$$

and bilinear fractional maximal operator

$$
B M_{\alpha}(f, g)(x)=\sup _{r>0} \frac{1}{(2 r)^{n-\alpha}} \int_{[-r, r]^{n}}|f(x-y) g(x+y)| d y .
$$


Also recall the following, less singular version of the bilinear fractional integral operator,

$$
\mathcal{I}_{\alpha}(f, g)(x)=\int_{\mathbb{R}^{2 n}} \frac{f(y) g(z)}{(|x-y|+|x-z|)^{2 n-\alpha}} d y d z, \quad 0<\alpha<2 n,
$$

and the associated maximal operator

$$
\mathcal{M}_{\alpha}(f, g)(x)=\sup _{Q \ni x}|Q|^{\frac{\alpha}{n}} f_{Q}|f(y)| d y \cdot f_{Q}|g(z)| d z .
$$

A similar calculation to that in the linear case shows that $B M_{\alpha}(f, g) \lesssim B I_{\alpha}(f, g)$ and $\mathcal{M}_{\alpha}(f, g) \lesssim \mathcal{I}_{\alpha}(f, g)$ when $f, g \geq 0$; moreover, it was shown in [24] (via $A_{\infty}$ extrapolation) that for $0<p<\infty$ and $w \in A_{\infty}$,

$$
\left\|\mathcal{I}_{\alpha}(f, g)\right\|_{L^{p}(w)} \leq C\left\|\mathcal{M}_{\alpha}(f, g)\right\|_{L^{p}(w)} .
$$

Here we use extrapolation to give a new proof of the following analogous inequality for $B I_{\alpha}$ and $M_{\alpha}$. This result was first proved in [25].

Theorem 4.7. Given $0<p \leq 1$ and $w \in R H_{\left(\frac{1}{p}\right)^{\prime}}$, then

$$
\left\|B I_{\alpha}(f, g)\right\|_{L^{p}(w)} \leq C\left\|\mathcal{M}_{\alpha}(f, g)\right\|_{L^{p}(w)} .
$$

Proof. Our proof is similar in parts to the proof of [25, Theorem 1.8], so we will only sketch the details. We will prove that the hypotheses of Theorem 1.1 are satisfied when $p_{0}=q_{0}=1$ : i.e., we will show that if $w \in R H_{\infty}$, then we have

$$
\int_{\mathbb{R}^{n}} B I_{\alpha}(f, g) w d x \lesssim \int_{\mathbb{R}^{n}} \mathcal{M}_{\alpha}(f, g) w d x
$$

for non-negative functions $f$ and $g$.

In $\left[25\right.$, Theorem 3.2] it was shown that $B I_{\alpha}$ is dominated pointwise by the dyadic operator

$$
B I_{\alpha}(f, g)(x) \lesssim B I_{\alpha}^{\mathcal{D}}(f, g)(x):=\sum_{Q \in \mathcal{D}} \frac{|Q|^{\frac{\alpha}{n}}}{|Q|} \int_{|y| \leq \ell(Q)} f(x-y) g(x+y) d y \cdot \chi_{Q}(x),
$$

where $\mathcal{D}$ is the standard dyadic grid. Now let $w \in R H_{\infty}$; then we estimate as follows:

$$
\begin{aligned}
\int_{\mathbb{R}^{n}} B I_{\alpha}^{\mathcal{D}}(f, g) w d x=\sum_{Q \in \mathcal{D}} & \frac{|Q|^{\frac{\alpha}{n}}}{|Q|} \int_{Q} \int_{|y| \leq \ell(Q)} f(x-y) g(x+y) w(x) d y d x \\
& \lesssim \sum_{Q \in \mathcal{D}} \frac{|Q|^{\frac{\alpha}{n}}}{|Q|}\left(\sup _{Q} w\right) \int_{Q} \int_{|y| \leq \ell(Q)} f(x-y) g(x+y) d y d x .
\end{aligned}
$$

If we make the change of variables $u=x-y, v=x+y$ and use the $R H_{\infty}$ condition on $w$, then

$$
\int_{\mathbb{R}^{n}} B I_{\alpha}^{\mathcal{D}}(f, g) w d x \lesssim \sum_{Q \in \mathcal{D}}|Q|^{\frac{\alpha}{n}}\left(f_{3 Q} f d x\right)\left(f_{3 Q} g d x\right)\left(\int_{Q} w d x\right) .
$$


This sum is similar to the sum that appeared in the proof of Theorem 4.5 except that it is over all dyadic cubes. However, we will bound it by a sum over a sparse family. Fix $a>1$; the exact value will be chosen later. Fix $k \in \mathbb{Z}$ and let

$$
\mathcal{C}^{k}=\left\{Q \in \mathcal{D}: a^{k}<\left(f_{3 Q} f d x\right)\left(f_{3 Q} g d x\right) \leq a^{k+1}\right\} .
$$

Let $\mathcal{S}^{k}$ be all cubes in $\mathcal{D}$ that are maximal with respect to inclusion and satisfy

$$
a^{k}<\left(f_{3 Q} f d x\right)\left(f_{3 Q} g d x\right) .
$$

(By an approximation argument we may assume $f$ and $g$ are bounded and have compact support, so such maximal cubes exist.) It is clear that every $Q \in \mathcal{C}^{k}$ is a subset of a unique cube in $\mathcal{S}^{k}$. We can now estimate the righthand side of inequality (4.4) as follows:

$$
\begin{aligned}
\sum_{Q \in \mathcal{D}}|Q|^{\frac{\alpha}{n}} & \left(f_{3 Q} f d x\right)\left(f_{3 Q} g d x\right)\left(\int_{Q} w d x\right) \\
& =\sum_{k \in \mathbb{Z}} \sum_{Q \in \mathcal{C}^{k}}|Q|^{\frac{\alpha}{n}}\left(f_{3 Q} f d x\right)\left(f_{3 Q} g d x\right)\left(\int_{Q} w d x\right) \\
& \leq \sum_{k \in \mathbb{Z}} a^{k+1} \sum_{Q \in \mathcal{C}^{k}}|Q|^{\frac{\alpha}{n}} \int_{Q} w d x \\
& =\sum_{k \in \mathbb{Z}} a^{k+1} \sum_{P \in \mathcal{S}^{k}} \sum_{Q \in \mathcal{D}(P)}|Q|^{\frac{\alpha}{n}} \int_{Q} w d x \\
& =\sum_{k \in \mathbb{Z}} a^{k+1} \sum_{P \in \mathcal{S}^{k}} \sum_{j=1}^{\infty} \sum_{Q \in \mathcal{D}(P)}|Q|^{\frac{\alpha}{n}} \int_{Q} w d x \\
& \lesssim \sum_{k \in \mathbb{Z}} a^{k+1}|Q|^{\frac{\alpha}{n}} \sum_{P \in \mathcal{S}^{k}} \int_{P} w d x \\
& \lesssim \sum_{Q \in \mathcal{S}}|Q|^{\frac{\alpha}{n}}\left(f_{3 Q} f d x\right)\left(f_{3 Q} g d x\right)\left(\int_{Q} w d x\right),
\end{aligned}
$$

where in the last line we let $\mathcal{S}=\bigcup_{k} \mathcal{S}^{k}$.

We claim that $\mathcal{S}$ is a sparse set. To see this, let

$$
\Omega_{k}=\bigcup_{Q \in \mathcal{S}^{k}} Q
$$

then $\Omega_{k} \supseteq \Omega_{k+1}$ and given $Q \in \mathcal{S}^{k}$ we have

$$
\left|\bigcup_{\substack{Q^{\prime} \in \mathcal{S} \\ Q^{\prime} \subsetneq Q}} Q^{\prime}\right|=\left|Q \cap \Omega_{k+1}\right| \leq\left|\left\{x: \mathcal{M}\left(f \chi_{3 Q}, g \chi_{3 Q}\right)(x)>a^{k+1}\right\}\right|
$$




$$
\leq\left[\frac{C}{a^{k+1}}\left(\int_{3 Q} f d x\right)\left(\int_{3 Q} g d x\right)\right]^{\frac{1}{2}} \lesssim \frac{C}{a^{\frac{1}{2}}}|Q| .
$$

The second inequality follows from the fact that $\mathcal{M}: L^{1}\left(\mathbb{R}^{n}\right) \times L^{1}\left(\mathbb{R}^{n}\right) \rightarrow L^{1 / 2, \infty}\left(\mathbb{R}^{n}\right)$. But then, if we choose $a$ sufficiently large, we get that $\mathcal{S}$ is sparse.

We can now complete the proof. Since $w \in R H_{\infty}$ we have $w(Q) \lesssim w\left(E_{Q}\right)$; since the sets $\left\{E_{Q}\right\}$ are disjoint,

$$
\begin{array}{r}
\sum_{Q \in \mathcal{S}}|Q|^{\frac{\alpha}{n}}\left(f_{3 Q} f d x\right)\left(f_{3 Q} g d x\right) w(Q) \lesssim \sum_{Q \in \mathcal{S}}|Q|^{\frac{\alpha}{n}}\left(f_{3 Q} f d x\right)\left(f_{3 Q} g d x\right) w\left(E_{Q}\right) \\
\leq \sum_{Q \in \mathcal{S}} \int_{E_{Q}} \mathcal{M}_{\alpha}(f, g) w d x \leq \int_{\mathbb{R}^{n}} \mathcal{M}_{\alpha}(f, g) w d x .
\end{array}
$$

If we combine the above estimates, we get the desired inequality and the proof is complete.

\section{REFERENCES}

[1] T. C. Anderson. A new sufficient two-weighted bump assumption for $L^{p}$ boundedness of Calderón-Zygmund operators. Proc. Amer. Math. Soc., 143(8):3573-3586, 2015.

[2] P. Auscher and J. M. Martell. Weighted norm inequalities, off-diagonal estimates and elliptic operators. Part I. General operator theory and weights. Adv. Math., 212(1):225-276, 2007.

[3] J. M. Conde-Alonso and G. Rey. A pointwise estimate for positive dyadic shifts and some applications. Math. Ann., 365(3-4):1111-1135, 2016.

[4] D. Cruz-Uribe, J. M. Martell, and C. Pérez. Extrapolation from $A_{\infty}$ weights and applications. J. Funct. Anal., 213(2):412-439, 2004.

[5] D. Cruz-Uribe, J. M. Martell, and C. Pérez. Weights, extrapolation and the theory of Rubio de Francia, volume 215 of Operator Theory: Advances and Applications. Birkhäuser/Springer Basel AG, Basel, 2011.

[6] D. Cruz-Uribe and C. J. Neugebauer. The structure of the reverse Hölder classes. Trans. Amer. Math. Soc., 347(8):2941-2960, 1995.

[7] D. Cruz-Uribe and C. Pérez. On the two-weight problem for singular integral operators. Ann. Sc. Norm. Super. Pisa Cl. Sci. (5), 1(4):821-849, 2002.

[8] A. Culiuc, F. Di Plinio, and Y. Ou. Domination of multilinear singular integrals by positive sparse forms. Preprint, 2016.

[9] J. Duoandikoetxea. Fourier analysis, volume 29 of Graduate Studies in Mathematics. American Mathematical Society, Providence, RI, 2001.

[10] J. García-Cuerva and J. L. Rubio de Francia. Weighted norm inequalities and related topics, volume 116 of North-Holland Mathematics Studies. North-Holland Publishing Co., Amsterdam, 1985.

[11] L. Grafakos and J.M. Martell. Extrapolation of weighted norm inequalities for multivariable operators and applications. J. Geom. Anal., 14(1):19-46, 2004.

[12] L. Grafakos and R. Torres. Maximal operator and weighted norm inequalities for multilinear singular integrals. Indiana Univ. Math. J., 51(5):1261-1276, 2002.

[13] E. Harboure, O. Salinas, and B. Viviani. Reverse-Hölder classes in the Orlicz spaces setting. Studia Math., 130(3):245-261, 1998.

[14] C. Hoang and K. Moen. Weighted estimates for bilinear fractional integral operators and their commutators. Indiana Univ. Math. J. to appear, 2016.

[15] T. Hytönen. The sharp weighted bound for general Calderón-Zygmund operators. Ann. of Math. (2), 175(3):1473-1506, 2012. 
[16] T. Hytönen, L. Roncal, and O. Tapiola. Quantitative weighted estimates for rough homogeneous singular integrals. Israel J. Math., 218(1):133-164, 2017.

[17] C. Kenig and E.M. Stein. Multilinear estimates and fractional integration. Math. Res. Let., 6(1):1-15, 1999.

[18] M. Lacey. An elementary proof of the $A_{2}$ bound. Israel J. Math., 217(1):181-195, 2017.

[19] A. Lerner, S. Ombrosi, C. Pérez, R. Torres, and R. Trujillo-González. New maximal functions and multiple weights for the multilinear Calderón-Zygmund theory. Adv. Math., 220(4):12221264, February 2009.

[20] A. K. Lerner. On an estimate of Calderón-Zygmund operators by dyadic positive operators. $J$. Anal. Math., 2012.

[21] A. K. Lerner and F. Nazarov. Intuitive dyadic calculus: the basics. Preprint, 2015.

[22] L. Liu and T. Luque. A $B_{p}$ condition for the strong maximal function. Trans. Amer. Math. Soc., 366(11):5707-5726, 2014.

[23] J. M. Martell and C. Prisuelos-Arribas. Weighted Hardy spaces associated with elliptic operators. Part I: Weighted norm inequalities for conical square functions. Trans. Amer. Math. Soc., 369(6):4193-4233, 2017.

[24] K. Moen. Weighted inequalities for multilinear fractional integral operators. Collect. Math., 60(2):213-238, 2009.

[25] K. Moen. New weighted estimates for bilinear fractional integral operators. Trans. Amer. Math. Soc., 366(2):627-646, 2014.

[26] C. J. Neugebauer. Inserting $A_{p}$-weights. Proc. Amer. Math. Soc., 87(4):644-648, 1983.

[27] C. Pérez. Two weighted inequalities for potential and fractional type maximal operators. Indiana Univ. Math. J., 43(2):663-683, 1994.

[28] C. Pérez. On sufficient conditions for the boundedness of the Hardy-Littlewood maximal operator between weighted $L^{p}$-spaces with different weights. Proc. London Math. Soc. (3), 71(1):135$157,1995$.

[29] M. M. Rao and Z. D. Ren. Theory of Orlicz spaces, volume 146 of Monographs and Textbooks in Pure and Applied Mathematics. Marcel Dekker Inc., New York, 1991.

[30] J. L. Rubio de Francia. Factorization and extrapolation of weights. Bull. Amer. Math. Soc. (N.S.), 7(2):393-395, 1982.

Theresa C. Anderson, Department of Mathematics, University of Wisconsin-Madison, MADISON, WI 53705, USA

E-mail address: tcanderson@math.wisc.edu

David Cruz-Uribe, OfS, Department of Mathematics, University of Alabama, Tuscaloosa, AL 35487, USA

E-mail address: dcruzuribe@ua.edu

Kabe Moen, Department of Mathematics, University of Alabama, Tuscaloosa, AL 35487, USA

E-mail address: kabe.moen@ua.edu 